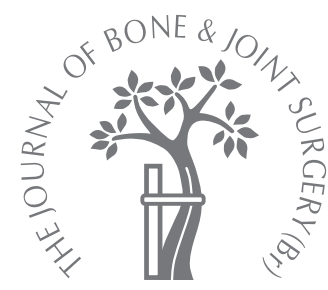

\title{
Lengthening of subscapularis and transfer of the lower trapezius in the correction of recurrent internal rotation contracture following obstetric brachial plexus palsy
}

\author{
J. A. Bertelli \\ From Governador \\ Celso Ramos \\ Hospital, \\ Florianópolis, Brazil
}

\begin{abstract}
An internal rotation contracture is a common complication of obstetric brachial plexus palsy. We describe the operative treatment of seven children with a recurrent internal rotation contracture of the shoulder following earlier corrective surgery which included subscapularis slide and latissimus dorsi transfer. We performed z-lengthening of the tendon of the subscapularis muscle and transferred the lower trapezius muscle to the infraspinatus tendon. Two years post-operatively the mean gain in active external rotation was $47.1^{\circ}$, which increased to $54.3^{\circ}$ at four years.

Lengthening of the tendon of subcapularis and lower trapezius transfer to infraspinatus improved the range of active external rotation in patients who had previously had surgery for an internal rotation contracture.
\end{abstract}

An internal rotation contracture of the shoulder is a common complication of obstetric brachial plexus palsy and may develop even in patients who make a full neurological recovery. ${ }^{1}$ In upper-type palsies the external rotators are paralysed, whereas internal rotators remain innervated. Either because of paralysis or late recovery of the external rotators, the unopposed subscapularis undergoes morphological changes, contracts ${ }^{2}$ and forces the humeral head posteriorly, thereby causing posterior dislocation and deformity of the glenoid.

Internal rotation contracture can be managed by bony or soft-tissue operations. In general, the former are preferred when the shoulder joint is incongruent, ${ }^{3}$ and such operations include external rotation osteotomy of the humerus or a combination of osteotomies of the clavicle, acromion and scapula. ${ }^{4}$ When joint deformities are absent, soft-tissue surgery is preferred and involves lengthening of the tendon of subscapularis or sliding the muscle from the scapula, as proposed by Pichon and Carlioz. ${ }^{5}$ Also, transfer of the latissimus dorsi has been used to enhance active external rotation, ${ }^{6}$ and the combination subscapularis slide and latissimus dorsi transfer has been popularised and evaluated by Pagnotta, Haerle and Gilbert. ${ }^{7}$

Recurrent internal rotation contracture was observed after Carlioz's procedure in 29 of 59 patients in Birch's series ${ }^{8}$ and after transfer of the latissimus dorsi recurrence of the deformity was observed in two of ten patients described by Al-Qattan. ${ }^{9}$ When Carlioz's procedure was combined with latissimus dorsi transfer, Phipps and Hoffer ${ }^{10}$ observed recurrence of internal rotation contracture in three of 35 patients, whereas Kirkos et $\mathrm{al}^{11}$ noted it in eight of their ten patients. The management of such patients has not yet received special attention in the literature, and other than bony procedures, ${ }^{4,12}$ further attempts at soft-tissue release have only been reported by Pearl et al. ${ }^{13}$

In this paper we describe our results with anterior subscapularis release ${ }^{8}$ combined with transfer of the lower trapezius muscle to infraspinatus in patients with recurrent internal rotation contracture after previous subscapularis release and latissimus dorsi transfer. We used the lower trapezius muscle because it is innervated by the accessory nerve, which is preserved in obstetric brachial plexus palsy, and in preliminary electromyographic studies on healthy volunteers we observed that this muscle is highly active during external rotation, making it suitable for transfer.

\section{Patients and Methods}

Seven children, with a mean age of seven years (4 to 9) were included in the study. All had an upper-type obstetric brachial plexus palsy and had previously undergone release of an internal rotation contracture using a subscapularis slide $^{5}$ and transfer of the latissimus dorsi muscle $^{6}$ at a mean age of 2.2 years (range 1.6 to 4). Whereas they all experienced initial improvement in abduction and external rotation, their shoulder function deteriorated 
Table I. Summary of patient data. The values in the post-operative period column, separated by commas, indicate results at the two- and four-year post-operative assessments

\begin{tabular}{|c|c|c|c|c|c|c|c|c|}
\hline \multirow[b]{3}{*}{ Patient } & \multirow{2}{*}{\multicolumn{2}{|c|}{ Age (yrs) }} & \multicolumn{4}{|c|}{ Active movement ${ }^{*}$} & & \\
\hline & & & \multicolumn{2}{|l|}{$\operatorname{ER}\left({ }^{\circ}\right)$} & \multicolumn{2}{|l|}{$\operatorname{Abd}\left({ }^{\circ}\right)$} & \multicolumn{2}{|c|}{ Trumpet sign $\left({ }^{\circ}\right)$} \\
\hline & First operation & $\begin{array}{l}\text { Second } \\
\text { operation }\end{array}$ & Pre-operative & Post-operative & Pre-operative & Post-operative & Pre-operative & Post-operative \\
\hline 1 & 4 & 9 & -40 & 20,10 & 45 & 50,50 & 90 & 10,10 \\
\hline 2 & 2 & 4 & -45 & 25,30 & 150 & 135,135 & 90 & 20,10 \\
\hline 3 & 2 & 5 & -20 & 0,25 & 90 & 90,90 & 70 & 10,10 \\
\hline 4 & 2 & 8 & -30 & 0,20 & 150 & 150,150 & 90 & 60,40 \\
\hline 5 & 1.6 & 5 & -20 & 30,30 & 160 & 150,150 & 90 & 10,10 \\
\hline 6 & 2 & 4 & -30 & 20,20 & 90 & 80,80 & 100 & 10,10 \\
\hline 7 & 2 & 7 & -20 & 30,40 & 90 & 50,50 & 50 & 10,10 \\
\hline
\end{tabular}

* ER, external rotation; Abd, abduction

and they developed a recurrent internal rotation contracture. All had equal restriction of active and passive external rotation. The former was measured with the elbow flexed to $90^{\circ}$ and passive limitation was examined with the elbow flexed and the shoulder adducted. The upper limit was defined as the point at which the first signs of resistance to passive external rotation became apparent and, thereafter, passive external rotation was at the expense of scapulothoracic movement. During this manoeuvre, the 'Putti sign'3 was seen in all cases. This consists of elevation of the superomedial angle of the scapula when the limb is adducted, the elbow flexed to $90^{\circ}$ and the shoulder externally rotated. The sign indicates contracture of subscapularis and posterior subluxation of the humeral head. ${ }^{3}$ The strength of internal and external rotation, with the elbow flexed to $90^{\circ}$ and the shoulder in a neutral position, was scored using the British Medical Research Council (MRC) scale. ${ }^{14}$

The pre-operative values for active external rotation and abduction are shown in Table I, along with the degree of shoulder abduction required when the hand was placed on the mouth (trumpet sign). On radiographic examination, all patients showed a simple posterior dislocation of the humeral head without abnormalities of the acromion or coracoid. $^{8}$

The author operated on all the patients between September 2000 and July 2004. The first had trapezius transfer 12 months after subscapularis release, and the remainder had both procedures during the same operation. The operations were performed under general anaesthesia. With the child supine, the upper limb was adducted, the elbow flexed and the shoulder externally rotated until scapulothoracic joint movement was observed. This manoeuvre was repeated after release of biceps and coracobrachialis and at the end of the operation, in order to confirm improvement in the range of external rotation. Through a deltopectoral incision, coracobrachialis and the short head of biceps were detached from the coracoid, the distal tip of which was removed to facilitate handling of the upper border of the subscapularis tendon which was z-lengthened. ${ }^{8}$ The limbs of the lengthened subscapularis muscle were sutured with the shoulder in maximal external rotation. The joint capsule was not divided. With the elbow flexed and shoulder externally rotated, the head of humerus, initially dislocated posteriorly, was reduced and movement of the humeral head from the dislocated position was easily felt during external rotation. With the head secured in the reduced position by the surgeon's hand, the elbow was flexed and the shoulder rotated internally. This confirmed that internal rotation was possible with the head in the reduced position.

The patient then was placed in a lateral decubitus position, with the affected arm uppermost. Through an incision over the spine of the scapula, the lower part of trapezius was detached from the inferior border of the spine of the scapula (Fig. 1). The tendon of trapezius was prolonged by dissecting its fascial attachments to the spine of the scapula as far as the acromion. The posterior deltoid was detached from the spine of the scapula and the tendon of infraspinatus dissected (Fig. 2). The lower aspect of the trapezius tendon was then sutured to the infraspinatus tendon under maximal tension with the shoulder adducted and in maximum external rotation (Fig. 3). The shoulder was immobilised in maximal external rotation and $30^{\circ}$ of abduction for three to four weeks.

Shoulder movement was assessed two and four years post-operatively by the author. The ranges of active and passive external rotation and abduction of the shoulder were measured, along with the degree of abduction needed for the patient to place their hand over their mouth. Also, the patients' capacity to touch their abdomen and back with the hand was appraised. The strength of internal and external rotation was tested manually and scored using the MRC scale. During the first post-operative year, physiotherapy and swimming were recommended, and radiographs were obtained to assess the position of the humeral head relative to the glenoid cavity.

Statistical analysis. Pre- and post-operative data on shoulder movement were analysed using repeated measures analysis of variance with Tukey's post test. All tests were twotailed. The threshold for statistical significance was set at $\mathrm{p}=0.05$. Because none of our patients had active external 


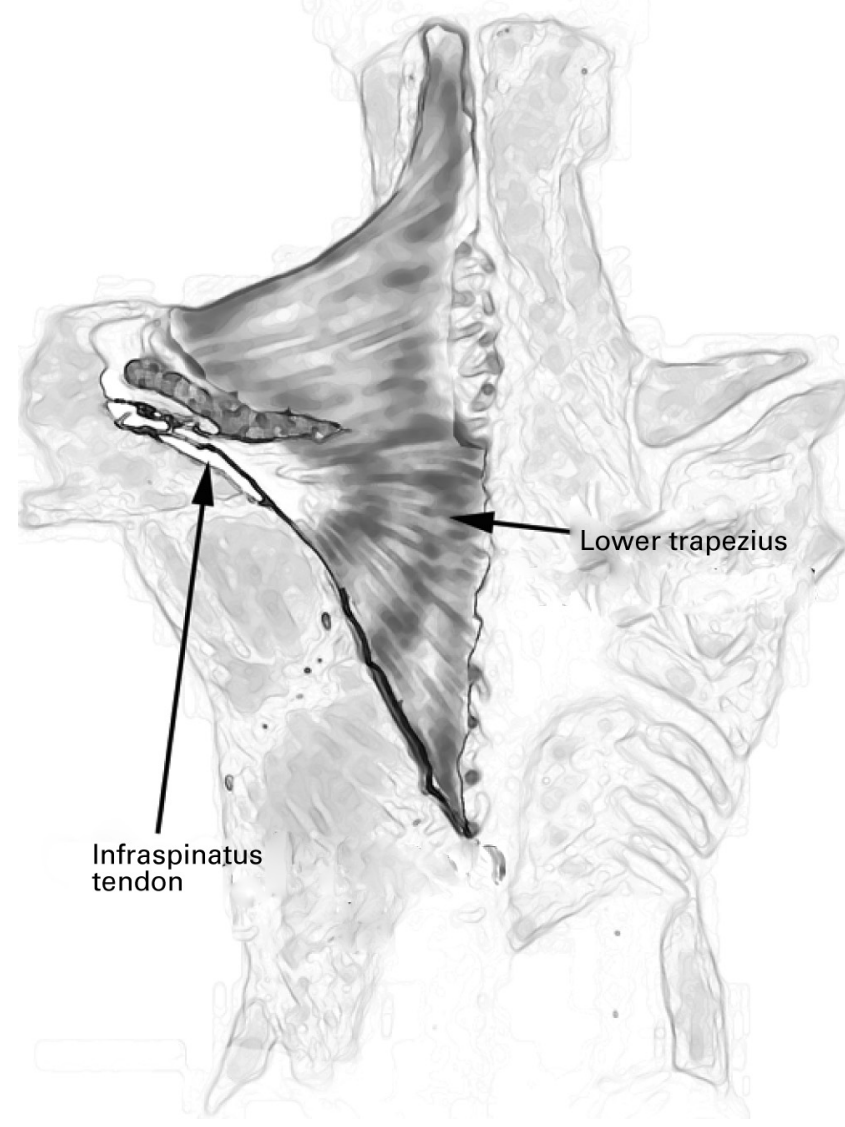

Fig. 1a

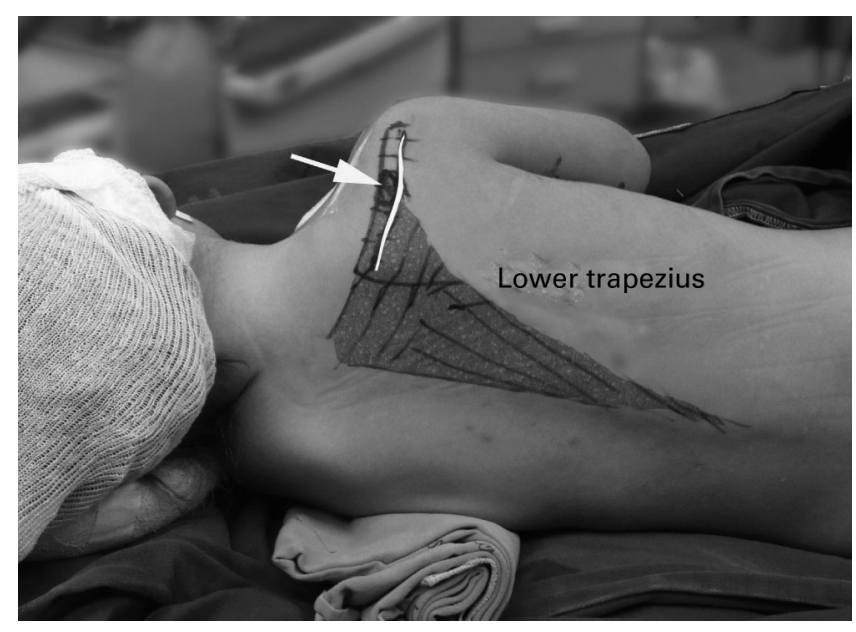

Fig. 1b

a) Schematic representation of the lower trapezius transfer and b) photograph showing position of the patient and incision for transfer of the lower trapezius to infraspinatus. The arrow indicates the tubercle on the spine of the scapula, where the lower trapezius is inserted. rotation pre-operatively the ranges of external rotation are expressed as negative values (Table I). For statistical evaluation, we considered parallel to the thorax from full internal rotation to be $0^{\circ}$ of external rotation, thereby allowing us to work with positive values.

\section{Results}

Following detachment of the short head of the biceps and corabrachialis and osteotomy of the coracoid, gains in external rotation were minimal $\left(<20^{\circ}\right)$ and noted in only four of the seven patients. External rotation was fully restored only after complete division of subscapularis, particularly its upper portion. After muscle division, external rotation allowed anterior translation of the head, thereby centring it on the glenoid. Conversely, internal rotation caused posterior dislocation of the head, and if the head was secured in the glenoid cavity by the surgeon's hand, full internal rotation was only possible with increased firmness, which induced additional scapulothoracic movement. Two years post-operatively, the mean gain in active and passive external rotation was $47.1^{\circ}\left(20^{\circ}\right.$ to $\left.70^{\circ}\right)$, increasing to $54.3^{\circ}$ $\left(50^{\circ}\right.$ to $\left.75^{\circ}\right)$ at four years. These values are statistically significant ( $\mathrm{p}=0.0004$ ) compared to their pre-operative state. However, the mean gain between two and four years was not statistically significant $(\mathrm{p}=0.06)$. Four children had improved movement between the two- and four-year assessment, but three were unchanged. In the post-operative period there was significant improvement in the trumpet sign $(\mathrm{p}=0.045)$, with complete resolution in six of the seven patients. External rotation strength was scored as M4 in two patients, M3 in one and $\mathrm{M} 3+$ in the remaining four. None had external rotation contractures or anterior instability of the shoulder joint (Table I). The range of active or passive abduction neither improved nor deteriorated postoperatively (Table I). Despite each patient being able to touch their abdomen and back, the final $20^{\circ}$ of internal rotation was scapulothoracic movement, as evidenced by elevation of the superomedial angle of the scapula, which we termed a 'reverse Putti sign'. This limitation in internal rotation was observed in five of the seven patients, but none was handicapped in daily activities. The strength of internal rotation was scored as M4 pre- and post-operatively in all patients. On axial radiographs the humeral head remained located in all cases and there were no abnormalities of the coracoid or acromion. The diameter of the humeral head and morphology of the glenoid cavity were not assessed because the contractures made it difficult to obtain adequate radiographs, especially pre-operatively. 


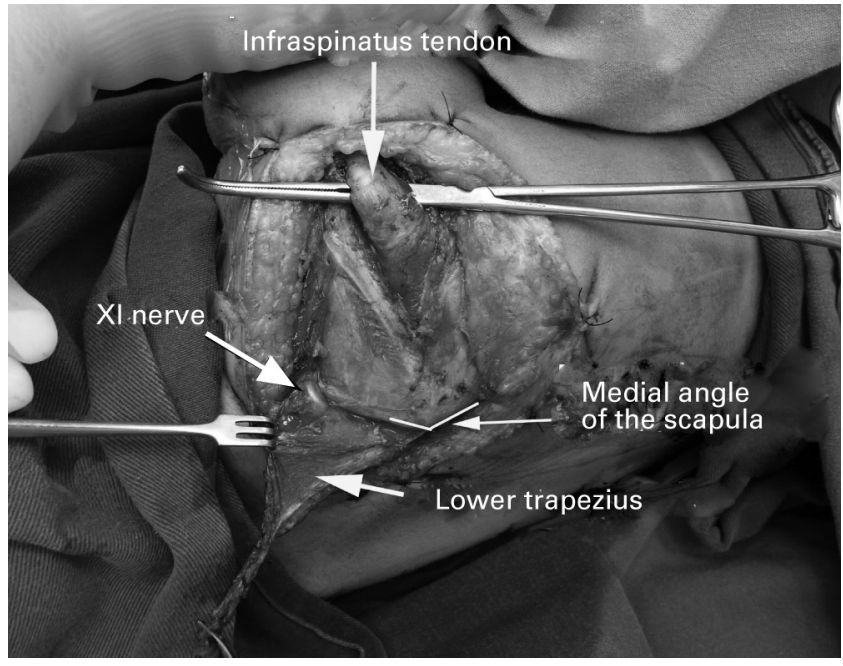

Fig. 2

Intra-operative view of the dissection of the lower trapezius muscle.

\section{Discussion}

In this study, we confirmed that subscapularis is the main block to external rotation in recurrent internal rotation contracture of the shoulder following obstetric brachial plexus palsy. It was not necessary to open the capsule or release the pectoralis major tendon. This concurs with the findings of Birch ${ }^{8}$ and Gilbert, ${ }^{14}$ but is contrary to the experience of Van der Sluijs et al, ${ }^{15}$ who needed to open the capsule in 15 of their 19 patients. We observed intraoperatively that, in most circumstances, undivided fibres in the upper subscapularis block full external rotation, and this should be evaluated carefully before a capsular incision is considered.

We believe that recurrent deformity is due to a new contracture of subscapularis, possibly because the transferred latissimus dorsi is ineffective as an external rotator.

We chose to release subscapularis through a deltopectoral approach which permits relocation of the humeral head and lengthening of the tendon of subscapularis. This could also have been accomplished arthroscopically, with the subscapularis tendon being sectioned rather than lengthened. ${ }^{13}$ This might adversely affect the strength of internal rotation. Because the humeral head was dislocated, there was no indication for a further subscapularis muscle slide.

Whereas Van der Sluijs et $\mathrm{al}^{15}$ reported severe external rotation contractures in almost half of their patients after Birch's procedure, we did not observe such a complication. Their contractures could have resulted from opening of the joint capsule, or prolonged post-operative immobilisation leading to adhesions around the coracoid. Price, Tidwell and Grossman, ${ }^{16}$ believe that immobilisation in excessive external rotation might overstretch the internal rotators, thereby contributing to an external rotation contracture.

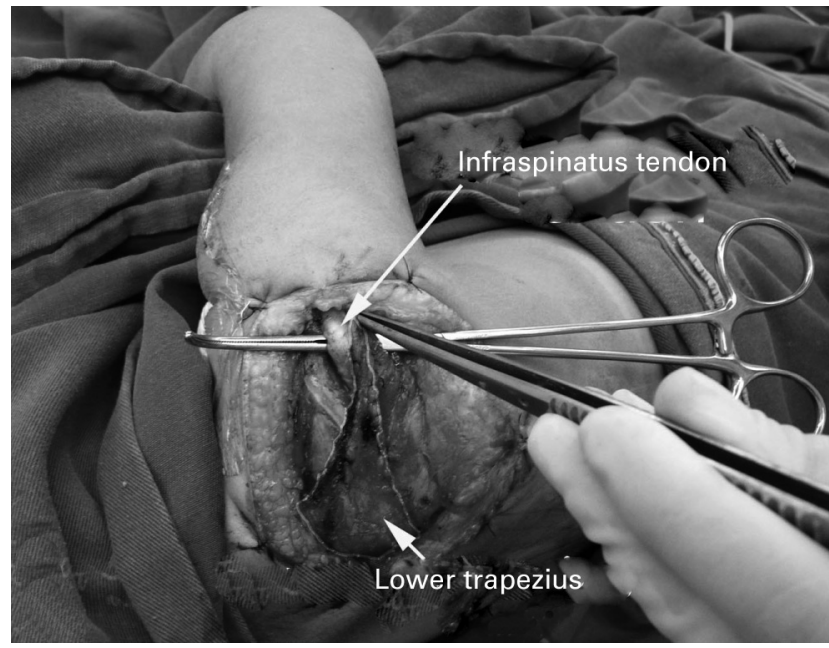

Fig. 3

Intra-operative view showing the lower trapezius is being prepared for suture to the infraspinatus tendon.

Internal rotation deficits have been reported regularly following subscapular release and shoulder relocation. Kambhampati et $\mathrm{al}^{17}$ observed a $10^{\circ}$ reduction in internal rotation, whereas a larger reduction of $42^{\circ}$ has been shown following arthroscopic tenotomy of the subscapularis tendon. ${ }^{13}$ In our patients, we anticipated about a $20^{\circ}$ loss and this difference might result from the greater pre-operative internal rotation contractures of our patients compared to Kambhampati's. ${ }^{17}$ Conversely, we observed a smaller loss of internal rotation than did Pearl et $\mathrm{al}^{13}$ which might be the effect of our preservation of subscapularis function by tendon lengthening rather than their tenotomy. In our series, the gain in external rotation largely exceeded any loss in internal rotaton. None of our patients was handicapped by their small limitation of internal rotation, and all compensated for it by scapular protraction and retraction and wrist flexion and extension. None complained of the deformity and we do not consider further surgical correction necessary.

The latissimus dorsi, whether accompanied or not by teres major, is the muscle usually transferred after shoulder release. However, latissimus dorsi is a strong internal rotator which, in brachial plexus injuries, might have been denervated and reinnervated; this may be achieved by foreign motor neurons. Therefore, natural antagonist function and aberrant reinnervation might contribute to the poor phasic conversion of the transferred muscle. Some surgeons believe that any benefit following latissimus dorsi transfer to the rotator cuff results solely from a tenodesis effect, which merely contributes to passive stabilisation of the humeral head. ${ }^{9}$ Also, when transferring latissimus dorsi, either alone or with teres major, the strength of internal rotation decreases. Therefore, initial improvement in external rotation might result simply from a decrease in strength of the antagonist. 
Moreover, as the latissimus dorsi is usually paralysed and unsuitable for transfer we believe that use of the lower trapezius to reinforce external rotation is promising, because its innervation from the accessory nerve is not affected in obstetric brachial plexus palsy; it is synergistic with external rotation, and internal rotation strength is not modified.

In the first patient the subscapularis was released and a year later a lower trapezius transfer was performed. There was initial improvement in passive external rotation, but not in active movement. This resolved after lower trapezius muscle transfer. After this experience shoulder release and lower trapezius transfer were performed at the same operation. Post-operatively there were contractions in the transferred lower trapezius during external rotation, and thus the transfer added stability. Moreover, improvement in external rotation from the second to fourth post-operative year was seen in four patients. Birch ${ }^{8}$ also observed improvements in external rotation after subscapularis lengthening without reinforcement of active external rotation by tendon transfer. However, his patients had not undergone previous surgery.

Our patients could have been treated by rotation osteotomy of the humerus, but this merely alters the arc of rotation to a more functional range ${ }^{18}$ and achieves an average improvement in external rotation of only about $25^{\circ}$, which is half the range we achieved. In favour of humeral osteotomy, however, is the possibility of increasing abduction by $20^{\circ}$ at least in the short term and in patients who had not undergone a previous soft-tissue procedure. ${ }^{12,18}$ Conversely, in our study we did not observe any improvement in abduction after lengthening of subscapularis and lower trapezius transfer, in agreement with the findings of Pearl et $\mathrm{al}^{13}$ after arthroscopic release and latissimus dorsi transfer. In our reconstructive strategy we prioritised external rotation over abduction, because the former is more important in daily activities, ${ }^{19}$ but there is no clear indication when soft-tissue release should be abandoned in favour of humeral osteotomy. The assumption that glenoid deformities and posterior dislocation preclude soft-tissue release is unsupported by this study, and accurate guidelines about which bony abnormalities preclude soft-tissue surgery need more detailed investigation. Nath and Paizi ${ }^{20}$ observed that scapular dysplasia and malposition contribute to impingement of the acromion and clavicle against the humerus, thereby preventing external rotation. They showed that levelling of the distal acromioclavicular triangle by multiple osteotomies and tightening the posterior glenohumeral capsule increased active external rotation by an average of $53^{\circ}$ in patients with obstetric brachial plexus palsy who had not undergone a previous soft-tissue procedure. ${ }^{4}$ Also, they reported improvements on the Mallet score ${ }^{14}$ in four patients who earlier had undergone soft-tissue release and humeral osteotomy. ${ }^{21}$ However, they failed to report any objective gain in external rotation.

Apart from the small number of patients and unblinded post-operative evaluation, a limitation of this study was the inability to determine remodelling of the shoulder joint accurately because a pre-operative internal rotation contracture prevented adequate radiographic evaluation. Also, the diameter of the humeral head is poorly evaluated in radiographic images. ${ }^{17}$ This shortcoming would have been overcome by MRI or CT. Also, although we did not report our outcomes using the Mallet scale, we believe this does not accurately reflect gains in external rotation or correction of the trumpet sign. Moreover, it is categorical and provides only ranks of freedom, which makes comparisons between studies difficult.

Further release of the subscapularis muscle by lengthening its tendon, combined with transfer of lower trapezius to infraspinatus, increases active external rotation in patients who have previously undergone surgery for a contractures due to obstetric brachial plexus palsy.

No benefits in any form have been received or will be received from a commercial party related directly or indirectly to the subject of this article.

\section{References}

1. Hoeksma AF, Ter Steeg AM, Dijkstra P, et al. Shoulder contracture and osseous deformity in obstetrical brachial plexus injuries. J Bone Joint Surg [Am] 2003;85A:316-22

2. Einarsson F, Hultgren T, Ljung BP, Runesson E, Fridén J. Subscapularis muscle mechanics in children with obstetric brachial plexus palsy. J Hand Surg Eur Vol 2008;33:507-12.

3. Zancolli EA, Zancolli ER. Chirurgie palliative dans le séeuelles de paralysie obstetricale. In: Tubiana R, eds. Traité de Chirurgie de la Main. Vol. 4. Paris: Edition, Masson 1991:635-58.

4. Nath RK, Lyons AB, Melcher SE, Paizi M. Surgical correction of the medial rotation contracture in obstetric brachial plexus palsy. J Bone Joint Surg [Br] 2007;89B:1638-44.

5. Pichon F, Carlioz H. Disinsertion of the subscapularis muscle in the treatment of obstetric paralysis of the upper limb. Chir Pediatr 1979;20:135-41 (in French).

6. Hoffer MM, Wickenden R, Roper B. Brachial plexus birth palsies: results of tendon transfers to the rotator cuff. J Bone Joint Surg [Am] 1978;60-A:691-5.

7. Pagnotta A, Haerle M, Gilbert A. Long-term results on abduction and external rotation of the shoulder after latissimus dorsi transfer for sequelae of obstetric palsy. Clin Orthop 2004;426:199-205

8. Birch R. Late sequelae at the shoulder in obstetrical palsy in children. In: Duparc J, ed. Surgical techniques in orthopaedics and traumatology, Vol. 3. Paris: Elsevier, 2003:1-7

9. Al-Qattan MM. Latissimus dorsi transfer for external rotation weakness of the shoulder in obstetric brachial plexus palsy. J Hand Surg [Br] 2003;28:487-90.

10. Phipps GJ, Hoffer MM. Latissimus dorsi and teres major transfer to rotator cuff for Erb's palsy. J Shoulder Elbow Surg 1995;4:124-9.

11. Kirkos JM, Kyrkos MJ, Kapetanos GA, Haritidis JH. Brachial plexus palsy secondary to birth injuries. J Bone Joint Surg [Br] 2005;87-B:231-5.

12. Kirkos JM, Papadopoulos IA. Late treatment of brachial plexus palsy secondary to birth injuries: rotational osteotomy of the proximal part of the humerus. J Bone Joint Surg $[A m]$ 1998;80-A:1477-83

13. Pearl ML, Edgerton BW, Kazimiroff PA, Burchette RJ, Wong K. Arthroscopic release and latissimus dorsi transfer for shoulder internal rotation contractures and glenohumeral deformity secondary to brachial plexus birth palsy. J Bone Joint Surg [Am]2006;88-A:564-74

14. Gilbert A. Early sequelae of the shoulder in obstetrical palsy. In: Duparc J, ed. Surgical techniques in orthopaedics and traumatology. Vol. 3. Paris: Elsevier, 2003:1-4.

15. Van der Sluijs JA, van Ouwerkerk WJ, de Gast $\mathbf{A}$, et al. Treatment of internal rotation contracture of the shoulder in obstetric brachial plexus lesions by subscapular tendon lengthening and open reduction: early results and complications. J Pediatr Orthop B 2004;13:218-24.

16. Price AE, Tidwell MA, Grossman JA. Is arthroscopic release indicated? J Bone Joint Surg [Am] 2007;89-A:452.

17. Kambhampati SB, Birch R, Cobiella C, Chen L. Posterior subluxation and dislocation of the shoulder in obstetric brachial plexus palsy. J Bone Joint Surg [Br]2006;88B:213-19 
18. Waters PM, Bae DS. The effect of derotational humeral osteotomy on global shoulder function in brachial plexus birth palsy. J Bone Joint Surg [Am]2006;88-A:1035-42.

19. Bertelli JA, Ghizoni M. Reconstruction of $C 5$ and $C 6$ brachial plexus avulsion injury by multiple nerve transfers: spinal accessory to suprascapular, ulnar fascicles to biceps branch, and triceps long or lateral head branch to axillary nerve. J Hand Surg [Am] 2004;29:131-9.
20. Nath RK, Paizi M. Scapular deformity in obstetric brachial plexus palsy: a new finding Surg Radiol Anat 2007;29:133-40.

21. Nath RK, Melcher SE, Paizi M. Surgical correcton of unsuccessful derotational humeral osteotomy in obstetric brachial plexus palsy: evidence of the significance of scapular deformity in the pathophysiology of the medial rotation contracture. J Brachial Plex Peripher Nerve Inj 2006;27:9. 